
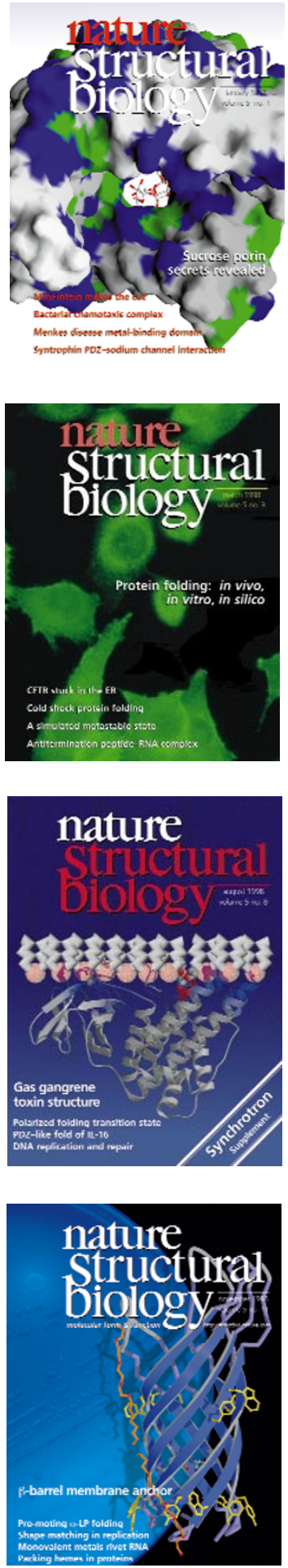

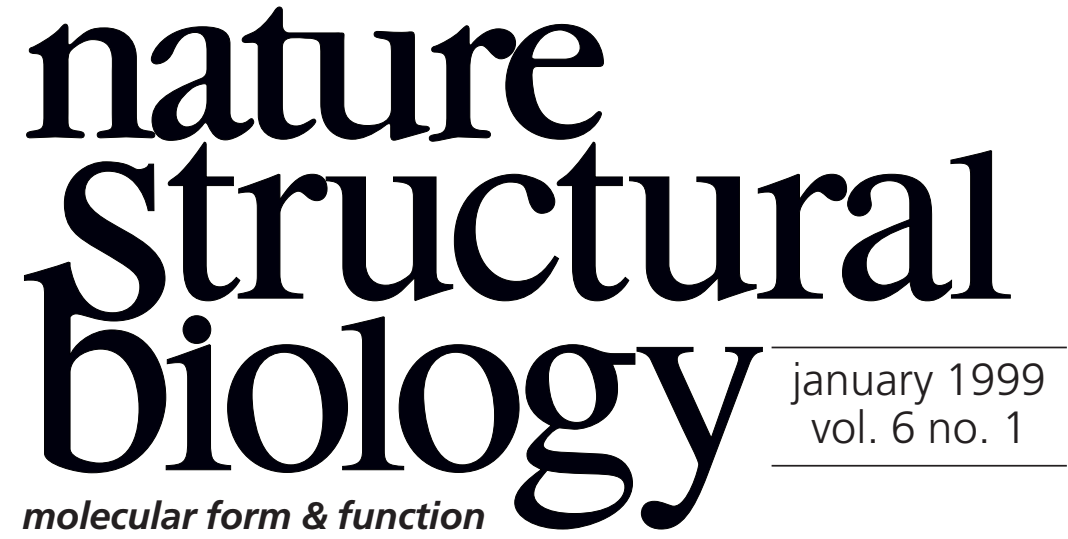

\section{A banner year for membranes}

Last year at this time, two Comments in Nature Structural Biology $y^{1,2}$ reflected on the need for funding agencies to support structural projects on membrane proteins - to move away from the so-called 'no crystals, no grant' funding rule. These Comments highlighted the fact that research on membrane proteins, which can be difficult to analyze by X-ray crystallography or NMR spectroscopy, generally lags behind research on soluble proteins. However, over the past year, many membrane protein structures have been determined and remarkable advances have occurred in several fields. This trend can be seen even in a survey of Nature Structural Biology papers featured on the journal's covers during 1998 - four of them (pictured here) illustrate either structural or biochemical studies on membrane proteins $s^{3-6}$. The current issue also focuses attention on membrane proteins - the cover depicts the structure of the FepA active transport protein ${ }^{7}$, and the Insight section presents progress on an algorithm for predicting transmembrane helix packing.

Unquestionably, one of the greatest advances of the past year — in any field, but especially in the area of membrane proteins - was the determination of the first structure of an ion channel, that of the KcsA potassium channel from the prokaryote Streptomyces lividans ${ }^{9}$ This structure (determined at a resolution of $3.2 \AA$ ) allows a detailed understanding of how these potassium channels prevent passage of other monovalent cations such as sodium. The structure suggests that sodium, which has an ionic radius that is $\sim 0.4 \AA$ smaller than that of potassium, is unable to interact efficiently with residues lining a portion of the channel known as the selectivity filter. This filter contains a number of polar residues that can accommodate only potassium ions as they lose their surrounding water molecules to pass through the channel. This study is remarkable for at least three reasons: (i) the structure was difficult to determine; (ii) it is a vivid visual manifestation of years of biophysical research; and (iii) it offers immediate insight into the ion channel's specificity. The function of the KcsA potassium channel was also furthered this past year by researchers who used electron paramagnetic resonance (EPR) spectroscopy to investigate its $\mathrm{pH}$-regulated gating ${ }^{10}$. Their work indicates that EPR spectroscopy can yield both static and dynamic structural information, even with membrane proteins that are traditionally difficult to analyze. These complementary studies, which were well worth the wait, have set the foundation for understanding other membrane proteins, such as voltage-gated ion channels.

Other long-awaited structures became available recently in the field of membrane fusion. Structures of proteins that participate in exocytosis or HIV infection have led to an enhanced knowledge of the mechanics required for fusion. Even though some of the proteins involved in these processes simply interact with transmembrane proteins and do not span membranes themselves, these collective studies represent one of the main highlights of membrane research from the past year. Now, it is possible examine the structure of the SNARE core complex $^{11,12}$, which must form prior to membrane fusion during exocytosis, and the structure of NSF ${ }^{13,14}$, which disassembles the SNARE complex after fusion. Using these structures as guides, detailed models for how fusion-active complexes assemble and are regulated can be developed and tested. Similarly, models for the HIV envelope glycoprotein, which is required for membrane fusion and infection, are developing as structures for its subunits become available. Most notably, the structure of the gp120 external envelope subunit, in complex with 
1. Martonosi, A.N. Nature Struct. Biol. 5 11-12 (1998).

2. Preusch, P.C. Norvell, J.C., Cassatt, J.C. \& Cassman, M. Nature Struct. Biol. 5, 12-13 (1998).

3. Forst, D., Welte, W., Wacker, T. \& Diederichs, K. Nature Struct. Biol. 5, 37-46 (1998).

4. Zhang, F., Kartner, N. \& Lukacs, G.L. Nature Struct. Biol. 5, 180-183 (1998).

5. Naylor, C.E. et al. Nature Struct. Biol. 5 738-746 (1998).

6. Pautsch, A. \& Schulz, G.E. Nature Struct Biol. 5, 1013-1017 (1998)

7. Buchanan, S.K. et al. Nature Struct. Biol. 6 56-63 (1999)

8. Pappu, R.V., Marshall, G.R. \& Ponder, J.W. Nature Struct. Biol. 6, 50-55 (1999).

9. Doyle, D.A. et al. Science 280, 69-77 (1998).

10. Perozo, E., Cortes, D.M., \& Cuello, L.G. Nature Struct. Biol. 5, 459-469 (1998).

11. Sutton, R.B., Fasshauer, D., Jahn, R. \& Brünger, A.T. Nature 395, 347-353 (1998)

12. Poirier, M.A. et al. Nature Struct. Biol. 5 765-769 (1998)

13. Lenzen, C.U., Steinmann, D., Whiteheart, S.W. \& Weis, W.I. Cell 94, 525-536 (1998)

14. Yu, R.C., Hanson, P.I., Jahn, R. \& Brünger A.T. Nature Struct. Biol. 5, 803-811 (1998) 15. Kwong, P.D. et al. Nature 393, 705-711 (1998).

16. Chan, D.C., Fass, D., Berger, J. M. \& Kim, P. S. Cell 89, 263-273 (1997).

17. Weissenhorn, W., Dessen, A., Harrison, $\mathrm{S}$ C., Skehel, J. J. \& Wiley, D. C. Nature 387 , $426-430$ (1997).

18. Caffrey, M. et al. EMBO J. 17, 4572-4584 (1998).19. Malashkevich, V. N., Chan, D. C., Chutkowski, C. T. \& Kim, P. Proc. Nat Acad. Sci. USA 95, 9134-9139 (1998).

20. Locher, K. et al. Cell 95, 771-778(1998).

21. Sali, A. Nature Struct. Biol. 5, 1029-1036 (1998)

22. Montelione, G.T. \& Anderson, S. Nature Struct. Biol. 6, 11-12 (1999).
CD4 and a neutralizing monoclonal antibody, was determined recently ${ }^{15}$. The gp120 subunit interacts with the primary HIV receptor CD4 and with a chemokine co-receptor to trigger membrane fusion, which is mediated directly by gp41, the HIV envelope glycoprotein transmembrane subunit. This gp120 structure, along with the fusion-active structures of the gp41 subunit that had been determined previously ${ }^{16-19}$, allows a better understanding of the events leading to HIV infection. Interesting similarities and differences between the two membrane fusion systems are now noticeable thanks to these advances. For example, both the SNARE core complex and the gp41 fusion-active structure are formed by coiled coils, but the nature and regulation of the structural arrangements are quite different in the two cases.

Recent research has also helped to redefine a 'typical' transmembrane protein. When many people think of membrane-spanning proteins, they think of $\alpha$-helical containing proteins, such as seven transmembrane receptors like rhodopsin. However, $\beta$-strands, forming a $\beta$-barrel motif, can also be used to anchor proteins to membranes. In fact, it appears that most (if not all) outer membrane proteins of bacteria such as Escherichia coli contain $\beta$-barrels. A recent paper in Nature Structural Biology presents an example of what is likely to be the smallest transmembrane unit composed of $\beta$-strands - the eight stranded E. coli outer membrane protein $\mathrm{A}(\mathrm{OmpA})$ transmembrane domain ${ }^{6}$. This small domain may be the $\beta$-barrel equivalent of a single $\alpha$-helical transmembrane anchor found in 'typical' membrane proteins. Now, at the other end of the spectrum, a paper on page 56 of this issue and a paper just published in Cell describe the structures of E. coli FepA ${ }^{7}$ and FhuA ${ }^{20}$, respectively, two related active transport outer membrane proteins that are both $\beta$-barrels composed of $22 \beta$-strands. These are the largest $\beta$-barrel structures yet determined. Does 8-22 $\beta$-strands constitute the full range of $\beta$-barrel sizes in membrane proteins? More membrane protein structures will be needed before this can be stated with certainty.

Improvements in techniques such as X-ray crystallography, NMR spectroscopy (both solution and solid state), EPR spectroscopy and electron crystallography will facilitate future structural studies on membrane-interacting proteins. Nevertheless, membrane protein research is likely to continue to lag behind studies of soluble proteins. This gap will likely be widened by structural genomics projects, which aim to determine the structures of predicted novel proteins. These efforts are focusing on 'high throughput' methods for solving structures of soluble proteins, and plans are underway to determine the structures of $\sim 10,000$ novel folds in the near future (ref. 21 and page 11 of this issue ${ }^{22}$ ). However, such an approach largely ignores membrane proteins, since they are less soluble. This is unfortunate, since integral membrane proteins constitute $15-25 \%$ of genomic sequences and $\sim 50 \%$ of receptors that are likely to be useful pharmaceutical targets. Although research on membrane proteins will undoubtedly benefit from technological advances made in the structural genomics efforts, proposals that focus solely on membrane protein research, such as the examples presented above, should continue to receive high priority (as the two Comments ${ }^{1,2}$ suggest) to increase knowledge about this important class of proteins.

\section{Meet the Editor}

The publishers are pleased to announce the appointment of Dr. Tracy Smith as Editor of Nature Structural Biology. Dr. Smith, formerly the Assistant Editor, succeeds Dr. Guy Riddihough, who resigned his position after five very successful years at the helm of Nature Structural Biology.

Nature Structural Biology was launched in January of 1994 and is now established as the leading journal devoted to structural research. As the field of structural biology has matured and expanded to include consideration of how molecular form affects function, so too has Nature Structural Biology expanded its interests ${ }^{1}$. Dr. Smith's broad scientific background and editorial experience make her well placed to continue this exciting development.

You are welcome to contact the Editor, email t.smith@natureny.com.

1. Editorial. Nature Struct. Biol. 5, 837-838 (1998). 